\title{
Periodicity of eating and human health: present perspective and future directions
}

Population dietary advice needs to be firmly rooted in cultural context. A very important part of that cultural context is the variation in food-consumption patterns evident within and between different populations. It follows that a knowledge of the factors influencing patterns of food consumption and of the impact of different patterns of food consumption on health is an essential element in devising culturally-appropriate dietary advice. The present workshop has, for the first time ever, brought together the many disciplines which study these factors: applied nutrition and dietetics, experimental nutrition, chronobiology, physiology, behavioural genetics, psychology, sociology, anthropology, clinical medicine and dentistry. Its achievement is in outlining our present state of knowledge and indicating how the diverse disciplines involved should interact in future research to fill the gaps in our present knowledge.

It is evident from the papers presented and from the extensive debate that there is a deficiency in quantifying periodicity of eating. It should be noted that the title of the workshop excludes any value-laden terms such as 'meals' or 'snacks'. Quantifying periodicity of eating in such terms will inevitably occur, but because such terms vary in their meaning between individuals, let alone between different cultures, their use should be limited in future research. Ingestive behaviour includes ingestion of foods, beverages and micronutrients. The definition of an eating occasion may vary in different studies, depending on the purpose of the investigation. However, it includes consideration of the minimal time span between intakes and the minimum level of intake. Thus, in future research on the periodicity of eating, an objective definition of what constitutes an eating occasion is needed. For example, for the study of energy intake an eating occasion may be defined as an event which provides at least $210 \mathrm{~kJ}$ with a separation in time from a preceding or follow eating event of at least $15 \mathrm{~min}$.

Another problem in quantifying periodicity of eating is one shared by all studies which seek to measure food and nutrient intake, namely inaccurate reporting. Given the wellestablished trend of the overweight to be particularly likely to under-report, much of the older cross-sectional epidemiological data, elegant for its day, linking hypercholesterolaemia, CHD, diminished glucose tolerance and obesity to reduced periodicity of eating, must be questioned. This is especially relevant to work on energy balance, given the evidence presented at the present workshop that the overweight are biased in underreporting of what are commonly referred to as 'snacks'. These methodological difficulties, therefore, necessitate cautious interpretation of the contribution of 'snacks' to nutrient intake, although on balance there are sufficient robust data to conclude that significant proportions of macronutrients and micronutrients are so derived.

A final point to be made in this context is that notwithstanding the difficulty of measuring periodicity of eating, the value of the data obtained should not be underestimated. There is an abundance of data on average daily intakes of nutrients but comparatively little knowledge regarding individual temporal distribution or of the interrelationships of such distributions between nutrients. Such knowledge would greatly help our understanding of the metabolic and behavioural effects of different periodicities of 
eating and would also sharpen our ability to design laboratory studies of the acute effects of single or multiple nutrient ingestions.

\section{Factors influencing eating}

Whilst a knowledge of the nature of variation in periodicity of eating is the bed-rock of this multi-disciplinary research area, the factors influencing this variation become the next level of study. The complexity of this task should not be underestimated. At the 'macro' level, there are cultural differences in periodicity of eating and at the 'micro' level, within given cultures sustained eating frequency patterns, as well as the amount and type of foods eaten, are influenced by food availability, cooking facilities, income, nature of employment, leisure activities including sports activities, mood, gender, age, education, geography etc. Short-term variation in the amount of food consumed is dramatically influenced by social facilitation. Surprisingly, recent data indicate that social facilitation appears to be affected more by genetic influences than by the previous childhood environment. Available data suggest that subsequent compensation occurs, without altered meal frequency, to keep energy intake constant. By contrast, established patterns of periodicity of eating will tend to be sustained in the face of short-term factors, such as social facilitation. In addition to these largely external factors, there is a complex web of largely internal physiological and psychological factors which greatly contribute to circadian and ultradian rhythms. Teasing out the exact contribution that these various external and internal cues make to periodicity of eating remains a challenge of considerable scholarly worth.

\section{Physiological and psychological effects of periodicity of eating}

Interest in the possible health benefits of modifying the periodicity of eating originates from epidemiological studies of the 1960s in which individuals consuming two or fewer 'meals' daily were shown to be significantly less healthy and more obese than those consuming five or more 'meals' daily. However, data presented at the workshop suggest that total energy expenditure is not influenced by the frequency of eating. Thus, the epidemiological association between eating frequency and body weight appears to be due either to an effect of eating frequency on energy intake, or to methodological errors resulting in spurious associations such as dietary under-reporting or alterations in eating patterns in response to weight gain (for example, missing meals in an attempt to lose weight). Although the latter explanation seems most likely, there are no prospective studies on the short- or long-term effects of altered periodicity of eating on overall energy intake.

Data presented at the workshop suggest that, in the short term, increasing the frequency of eating under controlled conditions, with no change in energy intake, results in acute reductions in postprandial blood glucose and insulin. At the present time, however, there is only one longer-term study in diabetic subjects and it showed that increased meal frequency had no effect on blood glucose or lipids. However, the study was not large enough to detect small effects and may have been confounded by a small increase in energy intake. In contrast, in normolipidaemic subjects, several short-term studies indicate that increased meal frequency with no change in energy intake or nutrient composition reduces serum cholesterol, but this has not been observed in hyperlipidaemic subjects. If the role of altering the periodicity of eating in the management of diabetes and hyperlipidaemia is to be determined, long-term studies need to be done, but these are likely to be costly and should be carried out by groups with experience in long-term dietary trials. 
Increased frequency of consumption of fermentable carbohydrates has a detrimental effect on dental health. However, this can be partly or completely offset by measures such as adequate fluoride intake and regular oral hygiene.

Little research has been undertaken on the effect of habitual patterns of food intake on physical performance. Research to date suggests that appropriate timing and amount of carbohydrate, before, during and immediately after exercise improves performance. There is clearly a need for more research in this area, both with respect to the influence of frequency of consumption and also the macronutrient profile of foods and drinks on physical performance.

Many factors affect the results of studies on the effects of diet on cognitive function and mood including the personality and beliefs of the subject, their level of arousal, the demands and nature of the task and their habitual dietary patterns. Most studies focus on the acute response to individual meals. It appears, for example, that eating breakfast or an afternoon snack improves cognitive function. Conversely, lunch appears to enhance the early afternoon dip in cognition. It is concluded that before the application of these findings it is essential that studies be performed on the chronic effect of the alteration of habitual eating patterns on cognitive function.

\section{Conclusions}

The recent Food and Agriculture Organization/World Health Organization (1996) report on the development of food-based dietary guidelines clearly requires that public health nutrition advice be rooted in cultural context. Available evidence from developed Western countries clearly indicate that, notwithstanding the perceived wisdom of the value of three 'square' meals daily, most people eat five to six times daily with a usual range of two to nine eating occasions daily. The deliberations of the present workshop would strongly suggest that this variation does not influence the balance of macronutrients or micronutrients consumed across the day. They also show that data relating variation in periodicity of eating to obesity is unconvincing.

Short-term studies show that more-frequent eating occasions may give rise to more favourable blood glucose metabolism and may also result in a reduced plasma LDLcholesterol. However, the longer-term effects have not been studied. The only potential deleterious effect of a high intake frequency is related to dental caries, which is preventable by good oral hygiene and fluoride exposure.

It also emerged during the course of the workshop that whilst it is possible to alter meal periodicity of eating in experimental settings, free-living subjects find long-term changes to their established eating frequency extremely difficult to maintain. Thus, at present, it is inappropriate to prescribe dietary advice based on any single ideal periodicity of eating.

\section{J. GiBNEY AND T. M. S. WOLEVER \\ SCIENTIFIC CO-CHAIRMEN}

\section{REFERENCES}

Food and Agriculture Organization/World Health Organization (1996). Preparation and Use of Food-based Dietary Guidelines. Report of a joint FAO/WHO consultation. Geneva: WHO. 